\title{
Antibody targeting facilitates effective intratumoral siRNA nanoparticle delivery to HER2-overexpressing cancer cells
}

\author{
Maria C. Palanca-Wessels ${ }^{1,2, *}$, Garrett C. Booth ${ }^{1}$, Anthony J. Convertine ${ }^{3}$, Brittany \\ B. Lundy ${ }^{3}$, Geoffrey Y. Berguig ${ }^{3}$, Michael F. Press ${ }^{4}$, Patrick S. Stayton ${ }^{3}$, Oliver W. \\ Press $^{1,3}$ \\ ${ }^{1}$ Clinical Research Division and Center for Intracellular Delivery of Biologics, Fred Hutchinson Cancer Research Center, \\ Seattle, WA, USA \\ ${ }^{2}$ Department of Medicine, Hematology Division, University of Washington, Seattle, WA, USA \\ ${ }^{3}$ Department of Bioengineering and Center for Intracellular Delivery of Biologics, University of Washington, Seattle, WA, USA \\ ${ }^{4}$ Department of Pathology, University of Southern California, Los Angeles, CA, USA \\ "This work was performed in Seattle, WA, USA. M.C.P.W. performed this work while at the Fred Hutchinson Cancer Research \\ Center, but is now employed by Seattle Genetics
}

Correspondence to: OliverW. Press, e-mail: press@u.washington.edu

Keywords: HER2 antibody, siRNA, targeted antibody delivery, ovarian cancer, breast cancer

Received: August 28, $2015 \quad$ Accepted: January 19, $2016 \quad$ Published: January 30, 2016

\section{ABSTRACT}

The therapeutic potential of RNA interference (RNAi) has been limited by inefficient delivery of short interfering RNA (siRNA). Tumor-specific recognition can be effectively achieved by antibodies directed against highly expressed cancer cell surface receptors. We investigated the utility of linking an internalizing streptavidinconjugated HER2 antibody to an endosome-disruptive biotinylated polymeric nanocarrier to improve the functional cytoplasmic delivery of siRNA in breast and ovarian cancer cells in vitro and in an intraperitoneal ovarian cancer xenograft model in vivo, yielding an $80 \%$ reduction of target $\mathrm{mRNA}$ and protein levels with sustained repression for at least $\mathbf{9 6}$ hours. RNAi-mediated site specific cleavage of target mRNA was demonstrated using the 5' RLM-RACE (RNA ligase mediated-rapid amplification of cDNA ends) assay. Mice bearing intraperitoneal human ovarian tumor xenografts demonstrated increased tumor accumulation of Cy5.5 fluorescently labeled siRNA and $70 \%$ target gene suppression after treatment with HER2 antibody-directed siRNA nanocarriers. Detection of the expected mRNA cleavage product by 5' RLM-RACE assay confirmed that suppression occurs via the expected RNAi pathway. Delivery of siRNA via antibody-directed endosomolytic nanoparticles may be a promising strategy for cancer therapy.

\section{INTRODUCTION}

The discovery of the RNA interference (RNAi) mechanism over a decade ago by Andrew Fire and Craig Mello stimulated intense interest in the potential application of short interfering RNA (siRNA), not only as a useful tool for the mechanistic study of cellular pathways but more significantly as a novel disease therapeutic. The ensuing years have resulted in a flurry of preclinical investigations and early phase clinical trials designed to test the utility of siRNA for the treatment of a variety of human diseases. $[1,2]$ In particular, siRNA for suppression of genes critical for tumor growth or resistance to chemotherapy may improve clinical outcomes and patient survival. The demonstration of the RNAi-mediated mechanism of target mRNA cleavage in human tumors from patients treated in Phase I clinical trials provides hope for clinical use of siRNA, however, the major obstacle of cell specific delivery remains to be overcome. $[3,4]$

The selective recognition of tumors by antibodies has been harnessed to directly elicit apoptosis, stimulate the immune-mediated clearance of cancer cells, or target cytotoxic agents to cancer cells. HER2 (also known as ERBB2 or NEU) is an internalizing cell 
surface receptor overexpressed or mutated in a variety of solid tumors including breast, gastric, lung and ovarian cancers. [5-9] Treatment with trastuzumab, a humanized mouse antibody directed against HER2, has become the standard of care for HER2-overexpressing breast cancer [6] and HER2-overexpressing metastatic gastric cancer. [10] HER2 has additionally been investigated as a portal through which receptor targeted antibodies or aptamers can deliver peptides, [11-13] siRNA [14, 15] or small molecule drugs [16] into cells. Recently, a drug conjugated form of trastuzumab (adotrastuzumab emtansine) that facilitates the intracellular delivery of the cytotoxic microtubule inhibiting agent mertansine was approved for the treatment of metastatic breast cancer. [17] Trastuzumab in combination with chemotherapy improved outcomes in gastric cancer patients, but single agent trastuzumab in ovarian cancer patients did not extend survival, although patients were selected for the clinical trial using relatively insensitive immunohistochemical assays without any confirmation of HER2 gene amplification status. [10, 18]

We previously developed a modular siRNA delivery system using a biotinylated endosome disrupting polymer that permits facile testing of combinations of antibody and siRNA and can be potentially tailored to various tumor types. [19] We hypothesized that a trastuzumab-directed siRNA nanoparticle could be used to enhance target gene suppression in HER2-overexpressing ovarian cancer cells. Ovarian cancer remains the most deadly cancer in women primarily due to its advanced state at diagnosis and rapid development of drug resistance. Little progress has been made over the past 20 years in improving the overall survival of patients, approximately $40 \%$ at 5 years, underscoring the need for novel therapeutic agents. [20]

Our group previously demonstrated the effectiveness of siRNA delivery to cells in vitro via a $\mathrm{pH}$-sensitive endosomolytic diblock co-polymer carrier bearing an internalizing antibody directed against the CD22 receptor expressed on lymphoma cells. [19] The linear carrier consists of: 1) a pH-responsive ampholyte block of poly(DMAEMA) (dimethylaminoethyl methacrylate), BMA (butylmethacrylate), and PAA (propylacrylic acid) groups; 2) a cationic poly(DMAEMA) block for binding siRNA; and 3) a terminal biotin to enable linkage to a streptavidin-conjugated monoclonal antibody (mAbSA). Electrostatic interactions promote complexation of siRNA to the polymeric micelles (Figure 1a). Targeted nanoparticles are formed by subsequent addition of $\mathrm{mAb}$ SA which attach to exposed surface biotin on micelles. Binding of antibody to cognate antigen stimulates receptor-mediated endocytosis and uptake into the tumor cell (Figure 1b). Subsequent protonation of PAA in the acidic environment of late endosomes induces a conformational change to predominantly hydrophobic unimers, disrupting the endosomal membrane and releasing siRNA into the cytoplasm. The modularity of this system permits testing of combinations of antibodies and siRNA customized to different tumor types. We sought to demonstrate the versatility and effectiveness of our polymer carrier system both in vitro and in vivo using the HER2 antibody, trastuzumab, in a solid tumor xenograft model of ovarian cancer.

\section{RESULTS}

\section{Intracellular uptake of nanoparticles by HER2- overexpressing cancer cells}

Binding and internalization of the trastuzumabpolymer siRNA nanoparticle was confirmed by flow cytometry in both HER2-overexpressing SKOV3 ovarian cancer and SKBR3 breast cancer cells using fluorescent AlexaFluor 647 labelled siRNA (Figure 2). Fluorescence intensity after 1 hour of incubation was markedly higher in cells exposed to nanoparticles targeted with streptavidinconjugated trastuzumab (Trast-SA) compared to nontargeted bovine herpes virus-1 antibody conjugate (BHV1$\mathrm{SA}$ ) or naked nanoparticles. Confocal microscopy of SKOV3 cells 24 hours after treatment with AlexaFluor 647 labeled siRNA showed a punctate pattern of fluorescence consistent with endocytic uptake (Figure 2). Enhanced uptake was similarly observed in SKOV3 cells using a different HER 2 antibody $10 \mathrm{H} 8$ which recognizes a separate epitope on the HER2 receptor (Supplementary Figure S1a).

\section{RNAi-mediated suppression via HER2 antibody- linked siRNA carrier}

Functional delivery was assessed in both HER2overexpressing ovarian and breast cancer cells using siRNA directed against the ubiquitously expressed glyceraldehyde3-phosphate dehydrogenase (GAPD) enzyme gene. Two hour pulse treatment of SKOV3 ovarian cancer cells with Trast-SA targeted nanoparticles containing GAPD siRNA resulted in greater reduction of GAPD expression at 48 hours compared to BHV1-SA as assessed by quantitative RT-PCR (Figure 3a) and GAPD enzyme activity (Supplementary Figure S1b). Verification of the RNAi mechanism of gene suppression was accomplished by the detection of the predicted 281 base pair fragment of GAPD mRNA using the 5' RLM-RACE assay (RNA ligase-mediated rapid amplification of cDNA ends) (Figure $3 b$ ). Sequencing of the isolated fragment verified that cleavage occurred at the expected site in the GAPD mRNA. Robust suppression of the GAPD gene was demonstrated for at least 96 hours after 2 hour pulse treatment of luciferase-expressing SKOV3 EA8 cells (Figure 3c). GAPD gene suppression was similarly demonstrated in the HER2-overexpressing breast cancer cell lines SKBR3 and BT-474 (Supplementary Figure S2). Treatment of cells with nanoparticles did not elicit cytotoxicity nor induce TLR-3 (toll-like receptor 3) activated immune response genes STAT1 (signal transducer 
and activator of transcription-1) or OAS1 (2'-5' oligoadenylate synthetase-1) genes (Supplementary Figure S3).

In order to demonstrate that suppression could be achieved using different clinically relevant siRNA sequences, we tested the functional delivery of siRNA designed against genes associated with chemotherapy resistance. High levels of $B C L-X L$ (B-cell lymphoma-extra large, also known as BCL2L1 or BCL2-like-1) are found in platinum resistant ovarian cancer cells and inhibition of $B C L-X L$ confers sensitivity to chemotherapeutic agents. [21, 22] STAT3 (signal transducer and activator of transcription-3) has been shown to upregulate $B C L-X L$ in solid tumors. [23] Significant suppression of $B C L-X L$ was demonstrated in SKOV3 EA8 cells pulse treated for

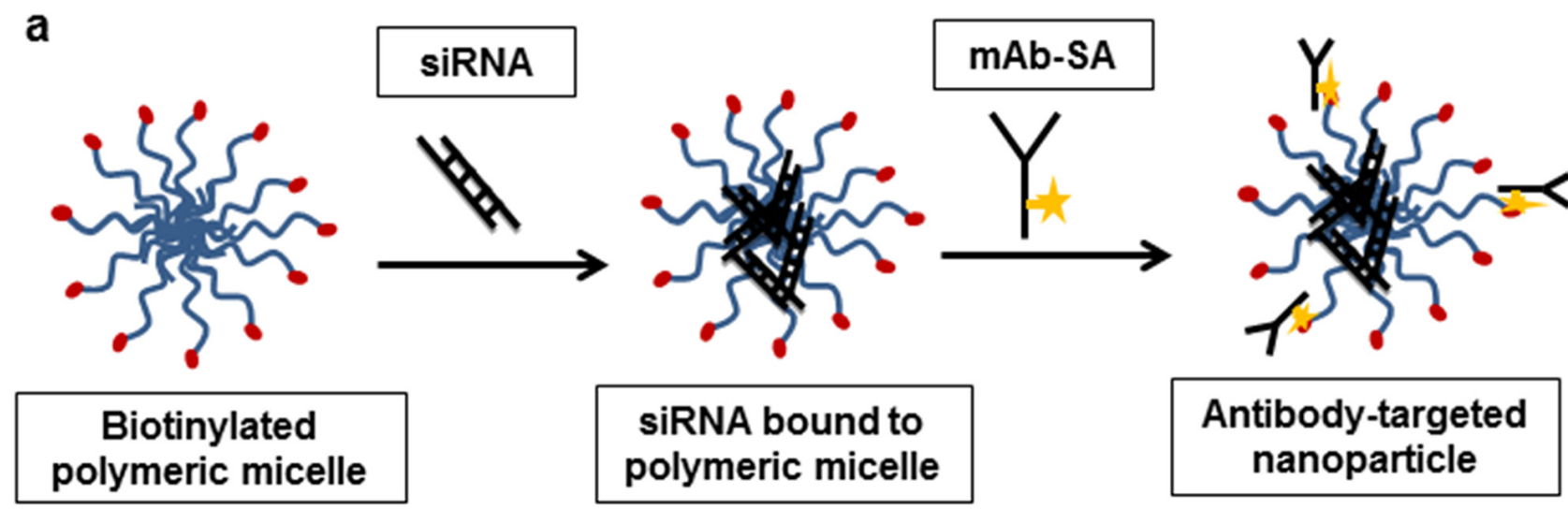

b

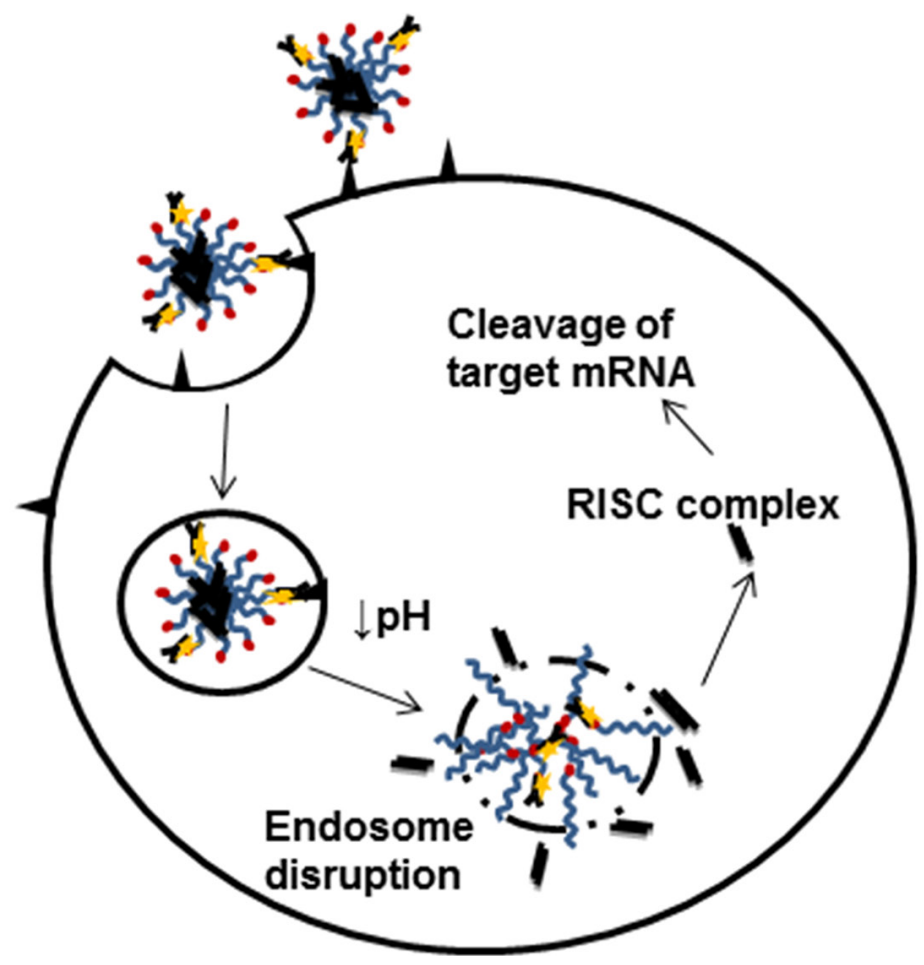

Figure 1: Antibody-targeted nanoparticle formation and intracellular siRNA delivery. a. The biotinylated diblock copolymer carrier consists of two modules: (1) a biotinylated cationic block comprised of poly(DMAEMA) and (2) an amphiphilic block consisting of DMAEMA, BMA and PPA groups. The linear polymer chains spontaneously form polymeric micelles with biotin exposed on the surface of the micelles. Polyplexes are formed by adding siRNA which associate with micelles via electrostatic interactions. Antibody-streptavidin conjugates (mAb-SA) are added and attach to available biotin moieties on the polymeric micelle surface resulting in antibody-targeted nanoparticles. b. Intracellular delivery is initiated by the recognition and binding of the antibody targeted nanoparticle to the cognate internalizing cell surface antigen on the surface of the tumor cell. Receptor-mediated endocytosis is triggered and the nanoparticle is internalized into the cell. As endosomal maturation occurs, the $\mathrm{pH}$ in the endosomal compartment drops and carboxyl groups in the amphilic block are protonated causing a conformational change of the polymer. This exposes the hydrophobic BMA side groups and disrupts the endosomal membrane leading to release of siRNA. siRNA is incorporated into the cytoplasmic RISC complex leading to catalytic cleavage of target mRNA and reduction of gene expression. 

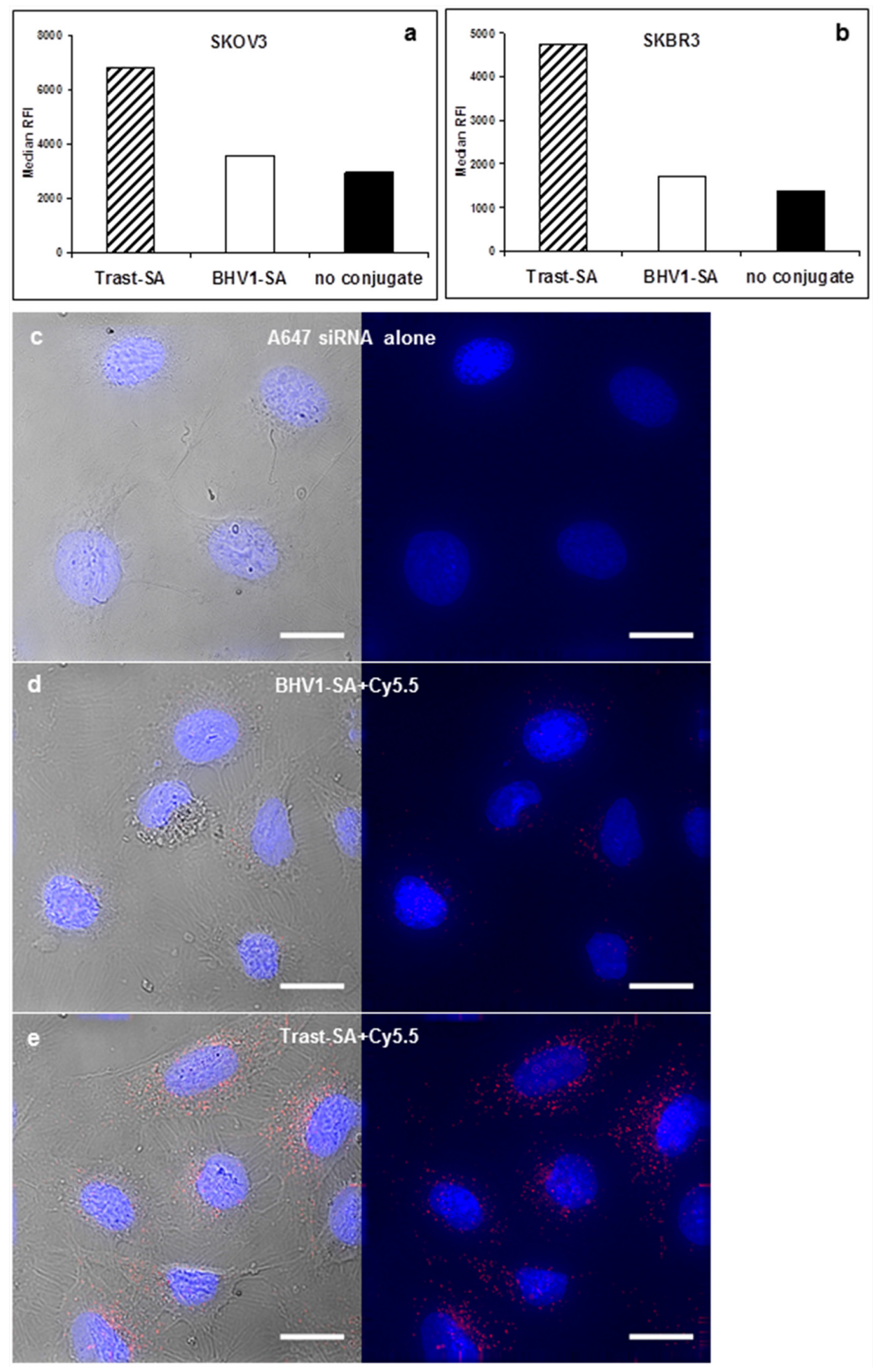

Figure 2: HER2 antibody conjugate Trast-SA enhances the uptake of siRNA-containing nanoparticles into HER2overexpressing SKOV3 ovarian and SKBR3 breast cancer cells. a. SKOV3 and b. SKBR3 cells were treated continuously for 1 hour with Trast-SA or BHV1-SA bearing nanoparticles or naked nanoparticles containing AlexaFluor 647 (AF647) labeled siRNA, trypsinized, then analyzed by flow cytometry for median relative fluorescence intensity (RFI). Representative results of 2 separate experiments are shown. c-e. SKOV3 cells grown on chambered glass slides were treated for 2 hours with (c) fluorescent AF647 labeled siRNA alone or contained within (d) BHV1-SA or (e) Trast-SA bearing nanoparticles prior to being rinsed and incubated with medium without nanoparticles. After 24 hours, cells were fixed, counterstained with mounting medium containing DAPI DNA stain, then visualized by fluorescence microscopy. Images were taken with a DeltaVision Elite wide deconvolution microscope at the original magnification of 80X. For each panel (c-e), both the fluorescence image (right) and fluorescence image merged with its respective differential interference contrast image (left) are shown. Scale bar equals 20 microns. 

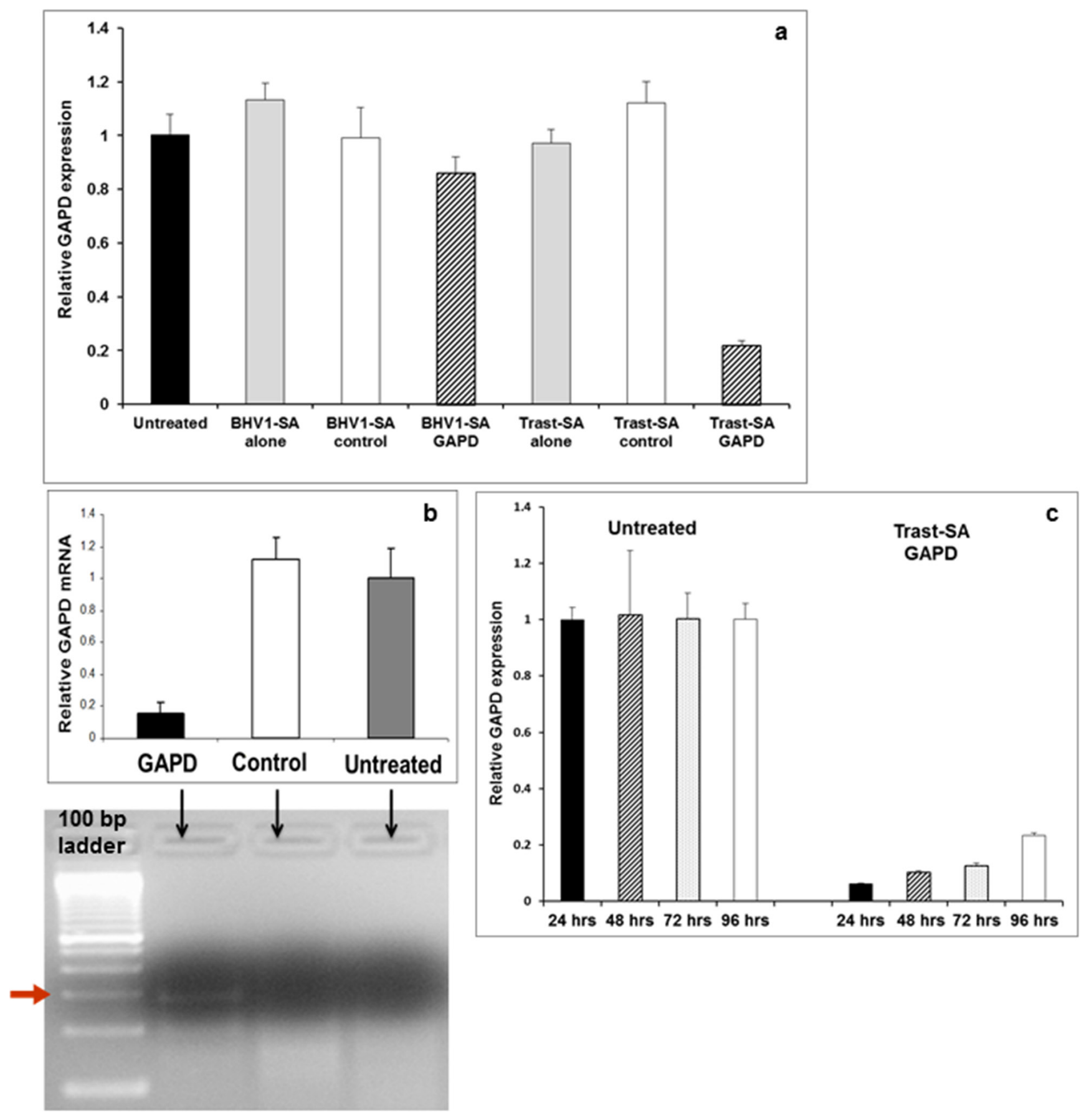

Figure 3: Suppression of GAPD gene expression by Trast-SA polymer mediated siRNA delivery in SKOV3 ovarian cancer cells. a. SKOV 3 cells were incubated for 2 hours in triplicate wells with GAPD or negative control siRNA contained in nanoparticles bearing Trast-SA or BHV1-SA conjugates or the respective antibody conjugates alone. Medium containing nanoparticles was then replaced with fresh medium alone and cells subsequently harvested 48 hours after the initial treatment and RNA extracted for qRT-PCR. Results are representative of three separate experiments. b. Verification of mRNA suppression via an RNAi mechanism was performed on SKOV3 cells that were pulse treated for 2 hours with Trast-SA bearing nanoparticles containing either GAPD or negative control siRNA and RNA extracted 48 hours later for qRT-PCR and 5'RLM-RACE analysis. The bar graph demonstrates reduction of target GAPD mRNA levels as measured by qRT-PCR in cells treated with GAPD but not control siRNA. Below the graph, gel electrophoresis of 5' RLM-RACE assay products from the respective treatments showed the predicted 281 base pair GAPD mRNA cleavage product only in the Trast-SA GAPD siRNA treated but not control siRNA or untreated cells supporting an RNAi mechanism for reduced GAPD expression. c. Sustained suppression of GAPD expression was observed in SKOV3 EA8 luciferase expressing cells treated for 2 hours with Trast-SA containing $G A P D$ siRNA then analyzed for GAPD mRNA level via qRT-PCR at various timepoints after treatment. 
2 hours with Trast-SA targeted nanoparticles containing $B C L-X L$ siRNA (Figure 4a) and showed an expected dose response with increasing siRNA concentrations (Figure 4b). The predicted 309 base pair mRNA cleavage product was demonstrated by the 5'RLM-RACE assay in cells treated with $B C L-X L$ siRNA but not negative control siRNA (Figure 4c). The mRNA cleavage product identity was verified by sequencing the cDNA band purified from the gel. Reduction of BCL-XL protein in cells was demonstrated by Western blot analysis (Figure 4d). Similar results were shown using a second $B C L-X L$ siRNA sequence (data not shown). Reduction of STAT3 mRNA was shown with qRT-PCR. Pretreatment with Trast-SA conjugate blocked HER2-mediated uptake of Trast-SA bearing nanoparticles containing STAT3 siRNA as demonstrated by reduction of STAT3 gene suppression to a level comparable to that achieved with non-targeting BHV1-SA conjugate (Figure 4e).

\section{Localization of siRNA to intraperitoneal ovarian tumor with HER2 antibody}

Ovarian cancer spreads locally within the abdominal cavity leading to tumor studding of peritoneal surfaces, blockage of lymphatic drainage and development of ascites. We used an intraperitoneal xenograft mouse model to recapitulate the typical clinical presentation in patients. Since assessment of intraperitoneal tumor burden by palpation is unreliable, we utilized the luciferaseexpressing SKOV3 clone EA8 to permit visualization of tumors in vivo with bioluminescence imaging. Mice bearing SKOV3 EA8 intraperitoneal ovarian tumors were imaged after luciferin injection by the IVIS Xenogen imaging system and separated into treatment groups with similar tumor burden as measured by average bioluminescence intensity. Mice were then injected with siRNA labeled with the near-infrared dye Cy5.5. Mice were sacrificed and organs were removed and imaged in an ex vivo fashion. We observed enhanced in vivo localization of siRNA delivered by trastuzumab-directed polymer to intraperitoneal ovarian tumors. Combined bioluminescence and fluorescence imaging after 24 hours revealed the accumulation of Cy5.5 siRNA in the kidneys whereas Cy5.5 siRNA administered within trastuzumabtargeted nanoparticles was still present in tumors at this timepoint (Figure 5a, 5b). Quantification of Cy5.5 was accomplished by homogenizing tissue and measuring the amount of Cy5.5 fluorescence relative to a standard curve generated by a known quantity of Cy5.5 labeled siRNA. This revealed a marked accumulation of Cy5.5 in tumors removed from mice treated with Trast-SA bearing siRNA nanoparticles compared to free siRNA. Tumors from mice treated with Trast-SA targeted nanoparticles contained an average of 228 picomoles of Cy5.5 per gram of tumor tissue compared to 11 picomoles in tumors from mice treated with non-targeted siRNA, $\mathrm{p}=0.005$ (Figure 5c).
Comparison of the amount of Cy5.5 in tumor and kidney showed an average tumor to kidney ratio of 0.6 versus $50(p=0.04)$ in mice treated with non-targeted siRNA or Trast-SA targeted siRNA respectively (data not shown). The average tumor to liver ratio of Cy5.5 was 1.8 versus $39(p=0.002)$ in mice treated with non-targeted siRNA or Trast-SA targeted siRNA, respectively (data not shown).

\section{Effective suppression of target genes in intraperitoneal ovarian tumors}

Functional delivery of GAPD siRNA to tumors in vivo was investigated. Mice bearing intraperitoneal SKOV3 EA8 tumors were divided into groups with a similar average tumor bioluminescence and treated with the following: PBS, Trast-SA alone, BHV1-SA targeted nanoparticles containing GAPD siRNA or Trast-SA targeted nanoparticles containing either control or GAPD siRNA. Mice were treated daily for two consecutive days at a dose of $4 \mathrm{mg} / \mathrm{kg}$ siRNA, then sacrificed 72 hours after the last dose. Intraperitoneal tumors were excised and RNA extracted. Reduction of GAPD gene expression by $70 \%$ with Trast-SA targeted nanoparticles containing GAPD siRNA was demonstrated via qRT-PCR compared to a $25 \%$ reduction with BHV1-SA non-targeted nanoparticles (Figure 6a). Additional mice were treated on the same schedule but at a dose of $2 \mathrm{mg} / \mathrm{kg}$ siRNA (Figures 6b, 6c). The 281 base pair predicted GAPD mRNA cleavage product was detected using the 5'RLMRACE assay in ovarian tumor tissue derived from mice treated with Trast-SA bearing nanoparticles containing GAPD siRNA but not in mice treated with Trast-SA nanoparticles containing negative control siRNA or TrastSA alone thus verifying RNAi-mediated GAPD gene suppression (Figure 6c). No induction of TLR3-activated immune response genes (OAS1 and STAT1) was detected (Supplementary Figure S4a, 4b). A minor three-fold increase in expression of mouse Ifit 1 (interferon-induced protein with tetratricopeptide repeats-1), another marker of immune response, was detected in the spleens of mice treated with siRNA containing nanoparticles bearing either Trast-SA or BHV1-SA (Supplementary Figure S4). No marked changes in pancreatic, hepatic, or renal function, nor elevation in creatinine kinase or glucose were detected between treatment groups (data not shown).

\section{DISCUSSION}

Effective intracellular delivery to target cells in vivo is undoubtedly the most difficult hurdle to overcome in the development of oligonucleotide therapeutics. Early phase clinical trials testing therapeutic siRNA in humans have shown favorable results, but it is still uncertain which delivery vehicle will ultimately prove to be the most effective for clinical application. We present encouraging preclinical data testing a modular antibody-directed 

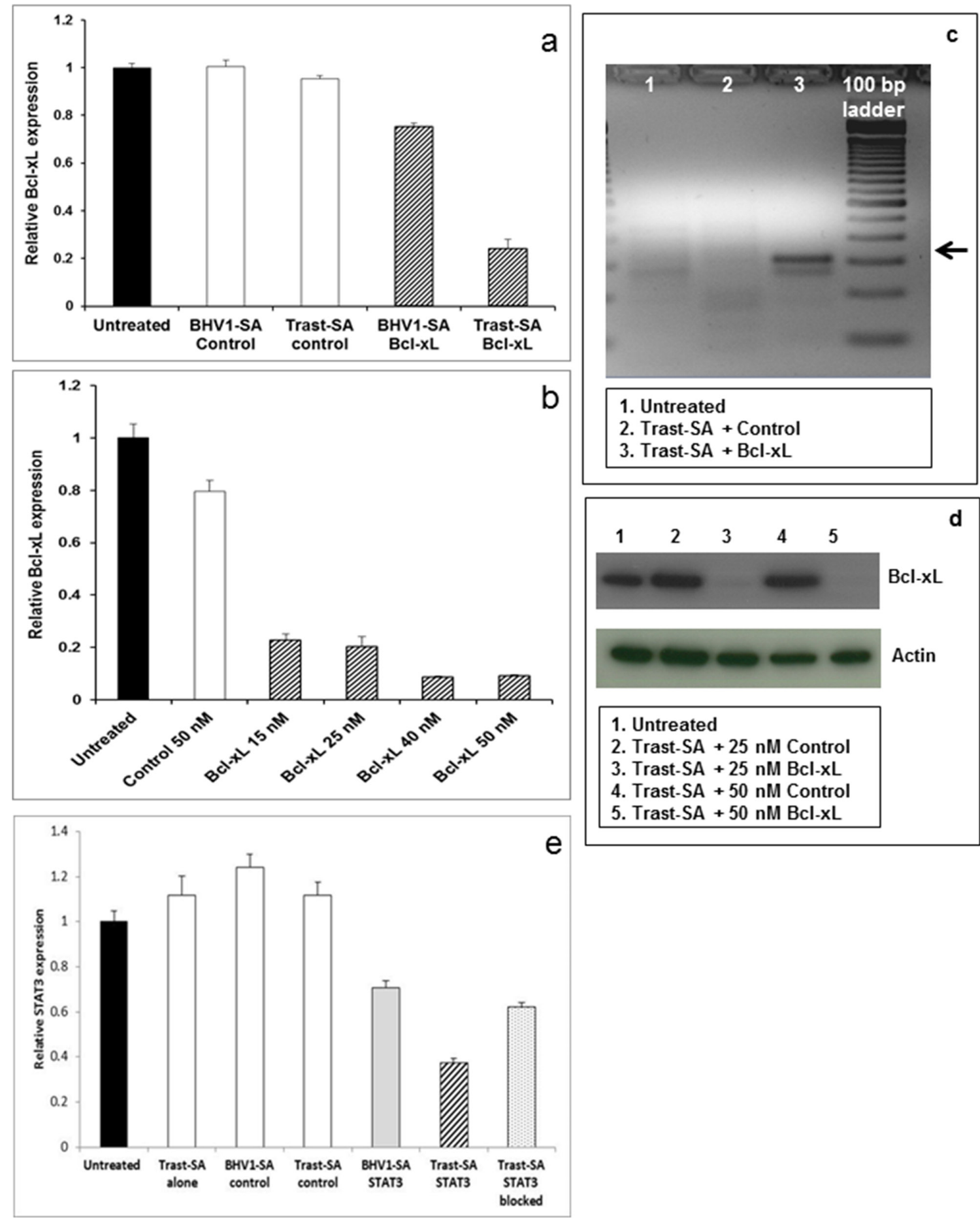

Figure 4: Effective suppression of oncogenes Bcl-xL and STAT3 in cancer cells. SKOV3 EA8 cells were treated for 2 hours with nanoparticles bearing Trast-SA or BHV1-SA conjugates and containing $25 \mathrm{nM}$ siRNA directed against $B C L-X L$ or a scrambled negative control bearing no homology to any known gene sequence. Cells were rinsed to remove medium containing nanoparticles and fresh medium introduced. After 48 hours, cells were harvested and RNA collected for a. qRT-PCR analysis and c. detection of the expected 309 base pair $B C L-X L \mathrm{mRNA}$ fragment (red arrow) by the 5'RLM-RACE assay. SKOV3 EA8 cells were similarly treated for 2 hours with Trast-SA targeted nanoparticles at a dose range ( 15 to $50 \mathrm{nM})$ of $B C L-X L$ siRNA or negative control siRNA at 25 or $50 \mathrm{nM}$. RNA or protein was collected at 48 hours for b. qRT-PCR analysis and $\mathbf{d}$. Western blot respectively. Results from cells treated with $25 \mathrm{nM}$ or $50 \mathrm{nM}$ negative control siRNA are shown since $B C L-X L$ expression levels did not vary by negative control siRNA dose. HER2 receptors on SKOV3 EA 8 cells were blocked by incubation with $37.5 \mathrm{nM}$ of Trast-SA alone for 30 minutes prior to 2 hour incubation with Trast-SA targeted polyplexes containing $25 \mathrm{nM}$ siRNA directed against STAT3. Other cells were treated with nanoparticles containing negative control or STAT3 siRNA bearing the indicated antibody conjugate targeting without preincubation. RNA was harvested 48 hours later and STAT3 levels assessed by qRT-PCR e. All qRTPCR analyses were performed for triplicate samples with standard deviation shown. Similar results were observed in duplicate experiments. 

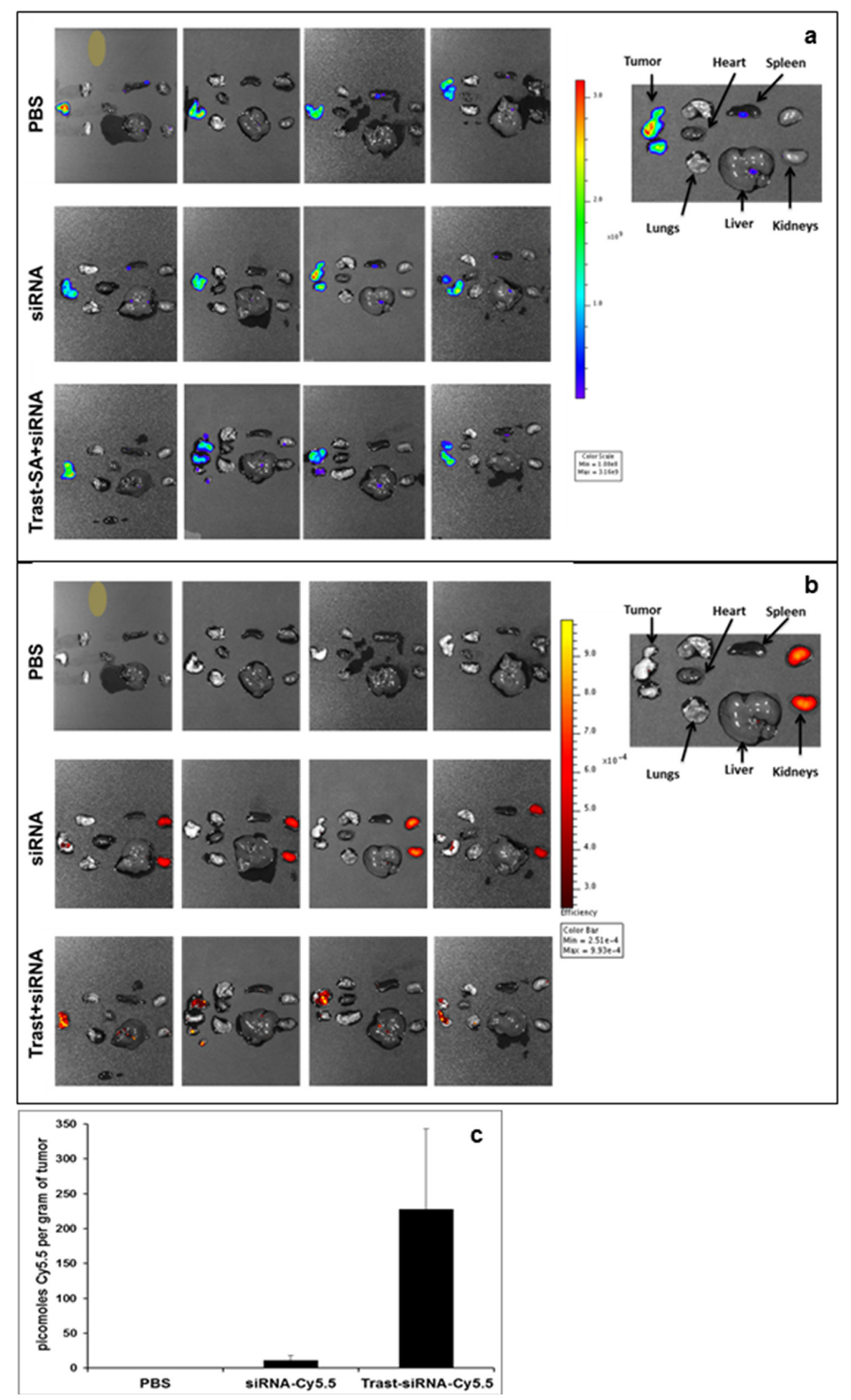

Figure 5: Intraperitoneal ovarian tumor localization of Cy5.5 fluorescently labeled siRNA after administration of HER2 targeted carrier. Athymic mice were injected intraperitoneally with SKOV3 EA8 luciferase expressing cells and tumor establishment verified by bioluminescence imaging using the Xenogen IVIS system. Two weeks after tumor cell injection, 4 mice per treatment group were injected with phosphate buffered saline (PBS), $2 \mathrm{mg} / \mathrm{kg}$ Cy 5.5 labelled siRNA alone or contained within TrastSA targeted nanoparticles. Twenty-four hours later, mice were injected with luciferin, euthanized, and organs (heart, lungs, liver, spleen, kidneys, tumor) removed. Tissues were imaged ex vivo for both a. bioluminescence to identify luciferase expressing tumor cells and $\mathbf{b}$. fluorescence to visualize siRNA biodistribution. c. Tumor tissue was weighed, homogenized in PBS and pelleted. Cy5.5 fluorescence intensity in the supernatant was measured at $675 \mathrm{~nm}$ excitation and $694 \mathrm{~nm}$ emission wavelength. The picomolar amount of Cy5.5 per gram in each tissue was determined by relating the measured fluorescence to a standard curve generated by known quantities of Cy5.5 siRNA. 

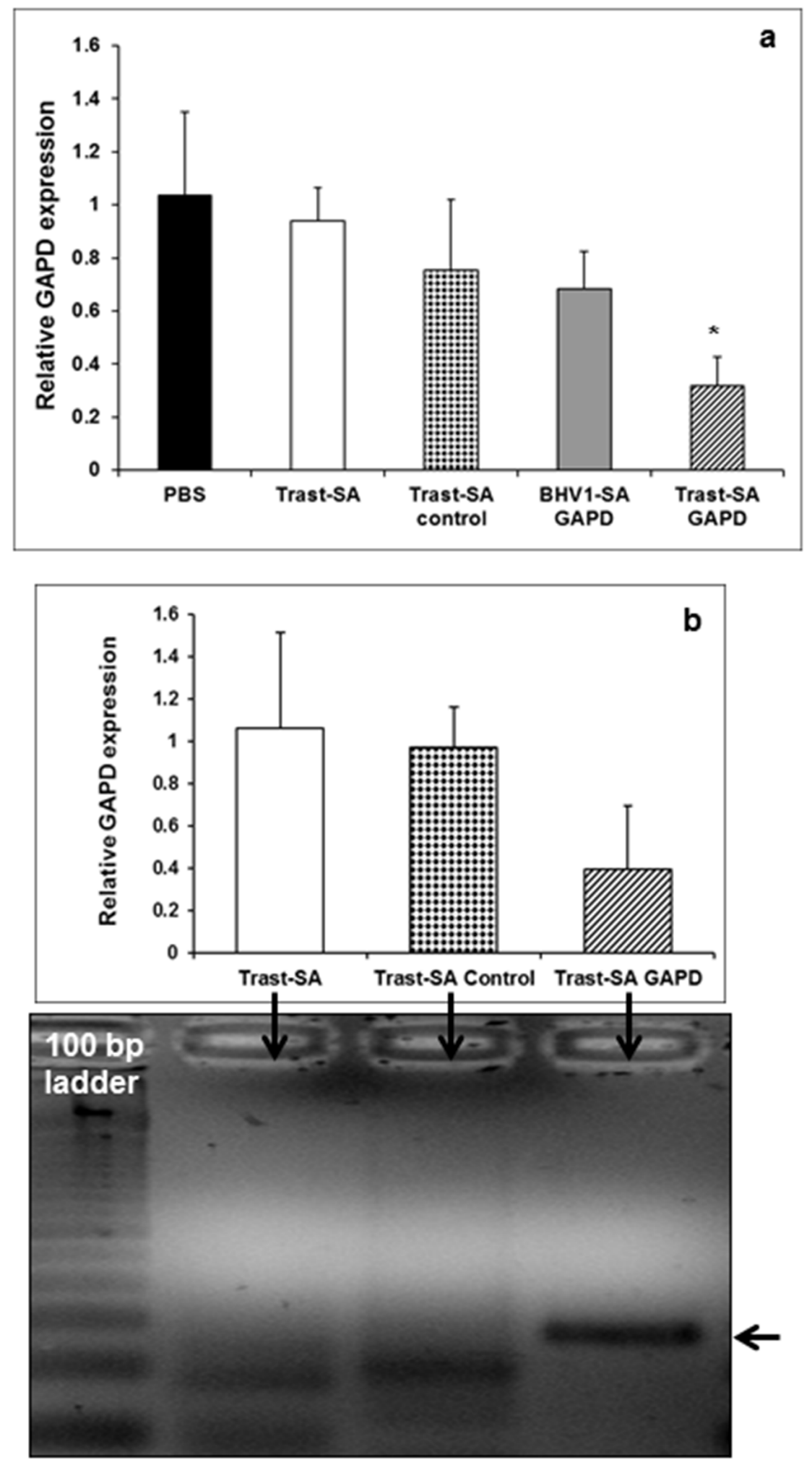

Figure 6: RNAi mediated target gene silencing within intraperitoneal ovarian cancer tumors after HER2 antibody directed siRNA delivery. Athymic mice bearing intraperitoneal SKOV3 EA8 tumors verified by bioluminescence imaging were treated with Trast-SA or BHV1-SA conjugate bearing nanocarriers containing negative control or GAPD siRNA, Trast-SA alone or PBS at doses of $\mathbf{a} .4 \mathrm{mg} / \mathrm{kg}$ ( $\mathrm{n}=5$ mice per group) or $\mathbf{b}, \mathbf{c} .2 \mathrm{mg} / \mathrm{kg}$ siRNA ( $\mathrm{n}=3$ mice per group) for two doses administered 24 hours apart. Mice were sacrificed 72 hours after the second dose and tumors collected for (a,b) qRT-PCR analysis and (c) detection of the 281 base pair GAPD mRNA cleavage product by 5'RLM-RACE assay. Error bars represent the standard deviation for qRT-PCR analyses. Asterisk indicates a significant difference $(\mathrm{p}<0.01)$ using the Student $t$-Test with one-tailed distribution between Trast-SA GAPD and BHV1-SA GAPD. 
biotinylated polymer carrier that can be easily adapted to different tumor types by varying the combination of antibody conjugate and target siRNA sequence.

We have demonstrated that this antibodydirected carrier can deliver functionally active siRNA to both ovarian and breast cancer cells overexpressing HER2. Two internalizing HER2 antibody conjugates (trastuzumab and 10H8) that recognize separate epitopes effectively facilitated intracellular delivery of siRNA. Non-specific limited uptake of particles bearing nontargeting BHV1-SA was observed and may be largely due to macropinocytosis which can be upregulated in cancer cells. [24] Blocking HER2 receptors with free Trast-SA conjugate prior to treatment with trastuzumabbearing nanoparticles reduced the level of suppression to that seen with non-targeting BHV1-SA, supporting the presence of an alternate pathway of uptake independent of HER2 receptor binding. The residual cationic charge on nanoparticles can promote non-specific association with negatively charged components on the cell surface membrane. Charge neutral carriers may reduce spontaneous non-targeted cellular uptake and our group is exploring alternative non-cationic carrier chemistries that utilize direct covalent disulfide linkage of siRNA to polymer rather than electrostatic interaction. [25] These neutral carriers are effective in vitro but require a higher siRNA concentration and longer continuous incubation time to achieve a level of gene suppression comparable to the antibody-targeted cationic diblock polymer used in the studies described in this report. Incorporation of an internalizing antibody or ligand moiety to the neutral siRNA carrier may counteract any loss of cellular uptake due to diminished charge interaction and improve the specificity of delivery and reduce the in vivo toxicity that can be observed with cationic carriers. Efforts to test this hypothesis are ongoing.

Verification of the intended mechanism of action is important in evaluating the efficacy of a therapeutic carrier. Detection of the predicted target mRNA cleavage product by $5^{\prime}$ RLM-RACE is the gold standard for confirmation of RNAi-mediated gene suppression. [26] We showed that the predicted mRNA cleavage products were detectable both by size on agarose gel electrophoresis and by sequencing of the PCR product using two different siRNAs designed against either $G A P D$ or $B C L-X L$ genes in cultured ovarian cancer cells. Furthermore, site specific cleavage of target mRNA was detected in mouse xenograft ovarian tumor tissue (Figure 6c) supporting the functional in vivo activity of siRNA delivered by HER2-targeted polymer carrier. Because activation of TLR3 by polymermediated siRNA delivery can lead to unintended effects from activation of the innate immune response, induction of OAS1 and STAT1 genes was assessed in tumors and found not to be upregulated in vivo. However, mouse Ifit 1 gene expression was increased approximately 3 to 4-fold in mouse spleen after treatment with Trast-SA or BHV1-SA targeted nanoparticles suggesting modest immune response activation. The significance of this low level of Ifitlupregulation is uncertain. Notably, siRNA treated mice exhibited no difference in behavior or appearance than mice receiving PBS alone. In comparison, a lipid-based carrier has been shown to induce a $247-$ fold increase of Ifit 1 mRNA expression and detectable serum interferon alpha levels in mice treated with $2 \mathrm{mg} /$ kg unmodified siRNA compared to PBS treatment. [27] Mitigation of the immune response may be achieved by use of 2'O-methyl modification of siRNA sequences [27] or pretreatment with dexamethasone. [28]

In conclusion, we have demonstrated the successful in vitro and in vivo antibody-targeted functional delivery of siRNA to HER2-overexpressing cancer cells. RNAimediated cleavage of target mRNA was verified as the mechanism of gene suppression. Antibody-targeted nanoparticle delivery of siRNA incorporating endosomolytic polymer carriers may be a useful strategy for cancer therapy. Similar targeted carriers to deliver small molecule drugs or peptides may also be promising. Future directions include testing therapeutic siRNA sequences both as monotherapy and in combination with chemotherapy with in vivo cancer models. Evaluation of other internalizing receptors expressed on a higher proportion of ovarian tumors than HER2 such as mesothelin is also of interest. [29]

\section{MATERIALS AND METHODS}

\section{Cell lines and media}

The SKOV3, SKBR3 and BT-474 cell lines were obtained from the American Type Culture Collection (ATCC; Manassas, VA). Authentication of the cell line was assured by the provider. All three of these cell lines have HER2 gene amplification and overexpression. Multiple aliquots were frozen from cells continuously passaged no longer than 3 months after initial receipt. Cells for experiments were passaged continuously for no longer than 3 months before being discarded and fresh aliquots resuscitated. The SKOV3 luciferase-expressing clone EA8 was generated by retroviral transduction of SKOV3 cells with a retrovirus encoding the firefly luciferase-Thy1.1Neo construct [30] followed by flow cytometric sorting for Thy 1.1 expression and selection of Thy 1.1 expressing cells with G418. SKOV3 EA8 was subsequently cloned by limiting dilution. All cell culture reagents were obtained from Invitrogen (Carlsbad, CA) except for fetal bovine serum (FBS) obtained from Thermo Scientific Hyclone (Logan, UT). SKOV3, SKOV3 EA8 and SKBR3 cells were grown in RPMI containing $10 \%$ fetal bovine serum, $100 \mathrm{IU} / \mathrm{ml}$ penicillin, $100 \mathrm{mcg} / \mathrm{ml}$ streptomycin, and 2 $\mathrm{mM}$ L-glutamine with the addition of $800 \mathrm{mcg} / \mathrm{ml} \mathrm{G} 418$ for the SKOV3 EA8 cells. BT-474 cell lines were grown in Hybri-Care Medium (ATCC) with 10\% FBS. All cells were maintained at $37^{\circ} \mathrm{C}$ in $95 \%$ air $/ 5 \% \mathrm{CO}_{2}$ incubator. 


\section{Polymer and antibody streptavidin conjugates}

Polymer was synthesized as previously described. [19] BHV1 (IgG1 anti-bovine herpes virus-1 antibody) was produced from a hybridoma obtained from ATCC and purified from ascites fluid over a HiTrap Protein G HP column (GE Healthcare; Piscataway, NJ). Trastuzumab used for experiments was clinical grade (Genentech; San Francisco, CA). 10H8 (an internalizing antibody to HER2) was produced in the laboratory of Michael F. Press (University of Southern California) as published. [31] Streptavidin conjugations to trastuzumab, BHV1 and 10H8 antibodies were performed by Donald K. Hamlin (University of Washington) using previously published techniques. [32,33]

\section{Polymeric micelle formation and transfection}

Polymeric micelles were reconstituted from lyophilized polymer to a concentration of $1 \mathrm{mg} / \mathrm{ml}$ or 10 $\mathrm{mg} / \mathrm{ml}$ in sterile PBS for in vitro or in vivo experiments respectively as previously described. [19] Polymer was added to siRNA at a polymer:siRNA molar ratio of $3: 1$ and incubated for 30 minutes at room temperature. The mAb-SA conjugates were added to polyplexes at 1:1 conjugate to available biotin molar ratios. Dynamic light scattering measurements of the polymeric micelles and siRNA complexes (N/P ratio $=3)$ in $150 \mathrm{mM}$ PBS at $\mathrm{pH} 7.4$ yielded hydrodynamic diameters of $34 \mathrm{~nm}$ and $37 \mathrm{~nm}$ respectively. The surface charge of the siRNApolymer complexes $(\mathrm{N} / \mathrm{P}$ ratio $=3)$, based on $\zeta$-potential measurements was found to be $4 \mathrm{mV}$, which suggests that particles are slightly positive under these conditions.

Cells were plated at a cell density of 50,000/ well in triplicate on the day prior to treatment. Cells were treated with polyplexes for 2 hours in medium containing 5\% FBS followed by a medium change to $10 \%$ FBS. Cells were harvested at 48 or 72 hours posttreatment for RNA and protein extraction respectively. The siRNA sequences were as follows: glyceraldehyde-3phosphate dehydrogenase (GAPD) (sense strand 5'-GGU CGGAGUCAACGGAUUUTT-3'; Integrated DNA Technologies; Coralville, IA); STAT3 (sense strand 5'-GCCUCUCUG CAGAAUUCAATT-3', Integrated DNA Technologies); $B C L-X L$ (sense strand 5'-GCUGGA GUCAGUUUAGAUGATT-3'; siRNA ID\# s1921; sense strand 5'-GGAACUCUAUGGGAACAAUTT-3'; siRNA ID\# s1922, Ambion); Ambion In Vivo GAPDH siRNA Cat\#4457291; Ambion In Vivo Bcl2L1 Cat\#4457308; Silencer Negative Control \#1 siRNA (Catalog AM4611; Ambion).

\section{Flow cytometry}

Cells were plated at 200,000/well in 12-well plates the day prior to treatment. Polyplexes were formed as above using AlexaFluor 647-labeled AllStars
Negative Control siRNA (\#1027287; Invitrogen) and added to cells in media containing 5\% FBS at a final concentration of $20 \mathrm{nM}$ of siRNA. After incubation at $37^{\circ} \mathrm{C}$ to allow internalization, cells were trypsinized and acid washed to remove residual surface bound nanoparticles and intracellular fluorescence measured on a BD FACS Canto flow cytometer with untreated cells set as a background reference as previously described. [19]

\section{Fluorescence microscopy}

SKOV3 EA8 cells were plated at 50,000/well in 2-well chamber slides and allowed to adhere overnight. Polyplexes were formed as above using AlexaFluor 647-labeled AllStars Negative Control siRNA (\#1027287; Invitrogen) and added to cells in media containing $5 \%$ FBS at a final concentration of $25 \mathrm{nM}$ of siRNA. After incubation at $37{ }^{\circ} \mathrm{C}$ for 2 hours, cells were rinsed to remove unbound nanoparticles and fresh medium added. After 24 hours, cells were rinsed three times in PBS, fixed with 10\% neutral buffered formalin for 15 minutes, and rinsed again in PBS prior to being coverslipped with Prolong Gold antifade reagent with DAPI (Invitrogen). Random fields were imaged at 60x (oil) with a DeltaVision Elite wide-field deconvolution microscope (Applied Precision, Issaquah, WA) fitted with a Photometrics HQ scientific grade cooled CCD camera and an Olympus 60×/1.42 Plan Apochromatic objective. Acquisition settings were constant for the AlexaFluor 647 signal. Three-dimensional data sets consisting of optical sections at 0.2 micron spacing were collected with the manufacturer's SoftWoRx software, and deconvolved using a constrained iterative algorithm. Data sets were processed to normalize intensities to the same scale using SoftWoRx software. Merging the data sets with their respective differential interference contrast (DIC) images was done with public domain software ImageJ.

\section{Quantitative RT-PCR}

RNA was isolated using the RNeasy mini kit (Qiagen; Valencia, CA) and reverse transcribed using TaqMan Reverse Transcription reagents (Applied Biosystems; Carlsbad, CA). PCR reactions were run in duplicate using an ABI Prism 7900HT real-time PCR instrument. Primers with FAM and VIC-labeled compatible probes for multiplex PCR were obtained from Applied Biosystems: GAPD (Catalog\#4352934E), peptidylprolyl isomerase A (PPIA) (Catalog\#4326316E), BCL-XL (Hs00236329_m1), STAT3 (Hs00374280_m1), STAT1 (Hs01013996_m1), OAS1 (Hs00973637_m1), mouse Ifit1(Mm00515153_m1). Relative quantification of gene expression was based on the reference value from the untreated control. [34] 


\section{5'-RLM-RACE and sequencing}

5' RLM-RACE was performed using the GeneRacer Kit (Invitrogen) with some modification as previously described. [35] Briefly, 100 ng total RNA was directly ligated to $250 \mathrm{ng}$ GeneRacer RNAOligo with T4 ligase. After phenol/chloroform extraction and ethanol precipitation, cDNA was synthesized using random primers. From this reaction, $1 \mu \mathrm{l}$ was used for first round 5'RACE reaction using the GeneRacer 5'primer and gene-specific primers (GAPD-specific reverse primer, 5'-CCTGCAAATGAGCCCCAGCCTTCTC-3' or BCL- $X L$ specific reverse primer, 5'-TCTACGCTTTCCACGCA CAGTGCCC-3') with the following cycling conditions: 1 cycle of $94{ }^{\circ} \mathrm{C}$ for 2 minutes, then 5 cycles of $94{ }^{\circ} \mathrm{C}$ for 30 seconds and $72{ }^{\circ} \mathrm{C}$ for 1 minute, then 5 cycles of 94 ${ }^{\circ} \mathrm{C}$ for 30 seconds and $70{ }^{\circ} \mathrm{C}$ for 1 minute, then 20 cycles of $94{ }^{\circ} \mathrm{C}$ for 30 seconds, and $68{ }^{\circ} \mathrm{C}$ for 1 minute. Secondround 5 ' RACE reaction was then performed using 1 $\mu \mathrm{l}$ of the first-round reaction and internal GeneRacer 5 ' nested and gene-specific primers (GAPD-specific nested, 5'-CGCCAGCATCGCCCCACTTGATTTT-3'or $B C L$ - $X L$-specific nested, 5'-GCTGTCCCTGGGGTGA TGTGGAGCT-3') using the above cycling conditions except for an extension time of 15 seconds and 25 cycles. PCR were performed using an Eppendorf Mastercycler thermocycler. PCR products were run on a 3\% agarose gel containing ethidium bromide then excised and extracted using a QIAquick Gel Extraction kit (Qiagen). Sequencing was performed using the ABI BigDye Terminator v3.1 Cycle Sequencing kit and subsequently analyzed on an ABI-3730xl DNA Analyzer (Applied Biosystems) per manufacturer's protocol.

\section{Immunoblotting}

Protein gel electrophoresis was performed by loading $5 \mu \mathrm{g}$ of cell lysate per lane on a 4-12\% Bis-Tris NuPAGE gel (Invitrogen) followed by transfer to a PVDF membrane (Invitrogen). The membrane was blocked with 5\% Milk/TBS $(0.1 \%$ Tween 20$)$, prior to overnight incubation with primary antibody incubation at $4^{\circ} \mathrm{C}$. Secondary antibody probing (Polyclonal Goat Anti-Rabbit Immunoglobulins/HRP; Dako; Carpinteria, CA) was done for 1 hour at room temperature. Results were visualized using Amersham ECL Western Blotting Detection Reagents (GE Healthcare; Pittsburg, PA) and Amersham Hyperfilm ECL (GE Healthcare). Reprobing to verify loading control was done after rinsing the membrane and reblocking before subsequent antibody probing. BCL-XL (clone 54H6), GAPD (clone 14C10), and beta-actin (clone 13E5) antibodies for immunoblotting were obtained from Cell Signaling Technologies (Danvers, MA).

\section{In vitro toxicity analysis}

Cells were plated in quadruplicate at a cell density of 5,000 cells/well in white 96-well plates on the day prior to treatment. Cells were treated with polyplexes for 2 hours then harvested 48 hours later and assayed using the CellTiter-Glo Luminescent Cell Viability Assay (Promega) per manufacturer's instructions. Microplates were read on a Centro LB 960 microplate luminometer (Berthold Technologies).

\section{GAPD protein assay}

GAPD enzyme activity was quantitated using the KDAlert GAPDH assay kit (Ambion) per manufacturer's recommendations. Cells were treated in quadruplicate then lysed 72 hours later. Lysate was transferred to a 96-well plate and KDAlert master mix added. The fluorescence intensity was measured over 4 minutes using an excitation wavelength of $560 \mathrm{~nm}$ and emission wavelength of $590 \mathrm{~nm}$.

\section{Xenograft experiments}

5-6 week old female athmyic $n u / n u$ mice weighing approximately 20 grams were obtained from Harlan Laboratories. Animals were housed in specific pathogenfree housing. All experiments were performed with the approval of the Fred Hutchinson Cancer Research Center Institutional Animal Care and Use Committee. 10 million SKOV3 luciferase-expressing cells suspended in sterile PBS (Gibco) were injected intraperitoneally. For imaging studies, mice were injected intraperitoneally with firefly D-luciferin (Biosynth International, Inc; Itasca, IL) at 150 micrograms per gram of body weight and tumor establishment was verified by in vivo bioluminescence imaging using the Xenogen Spectrum system (Perkin Elmer; Waltham, MA). Mice were separated into treatment groups with similar average bioluminescence intensity and then injected intraperitoneally with Cy5.5-labeled siRNA alone, a trastuzumab-targeted polyplex containing Cy5.5labeled siRNA, or sterile PBS alone. After 24 hours, mice were euthanized via carbon dioxide inhalation. Organs were removed and imaged in an ex vivo fashion. Kidney, liver, and tumor nodules were flash frozen for further analysis. For functional siRNA delivery experiments, mice were injected intraperitoneally with 2 or $4 \mathrm{mg} / \mathrm{kg}$ siRNA once daily for two days then euthanized via carbon dioxide inhalation 72 hours after the second dose. Blood for laboratory toxicity analysis was harvested via cardiac puncture. Tumor and spleen were placed in RNAlater (Qiagen) for RNA extraction for qRTPCR and 5'RACE analysis (tumor only).

\section{RNA extraction and quantitation from tissue}

RNA was extracted from mouse tissues as previously described. [36] Briefly, samples were homogenized in guanidine thiocyanate lysis buffer with the FastPrep-24 instrument (MP Biomedicals; Solon, OH) and Lysing Matrix D tubes (MP Biomedicals). Samples were immediately centrifuged at $4{ }^{\circ} \mathrm{C}$, extracted with phenolchloroform and RNA precipitated with isopropanol and 
sodium acetate (Ambion) overnight at $-20^{\circ} \mathrm{C}$. Further RNA purification was performed using the RNeasy kit (Qiagen; Valencia, CA) per manufacturer's instructions after adding $350 \mu \mathrm{l}$ RNEasy RLT buffer and $100 \mu \mathrm{l} 100 \%$ ethanol to the resuspended RNA pellet. Residual DNA contamination was removed with the Turbo DNA-Free kit (Ambion). The supernatants were collected and quantitated via UV-Vis for concentration analysis.

\section{Biodistribution}

Quantification of Cy5.5-labeled siRNA in tissues was performed as previously described. [37] Briefly, tissue was homogenized in PBS with the FastPrep-24 instrument and Lysing Matrix D tubes. Homogenates were centrifuged to remove insoluble tissue debris. The fluorescence intensity of the supernatant was analyzed using a Synergy H4 microplate reader (BioTek; Winooski, VT) at 675 and $694 \mathrm{~nm}$ of excitation and emission wavelength, respectively. The moles of siRNA per gram of tissue was determined by using a standard curve generated by diluting $0.5 \mu$ moles of Cy5.5-labeled GAPD siRNA 1:2 in PBS in succession six times. Tissue samples were diluted 1:2 in PBS before being read in duplicate along with the standards and a blank PBS sample.

\section{ACKNOWLEDGMENTS}

We thank Shani Frayo and Aimee Kenoyer (Fred Hutchinson Cancer Research Center, FHCRC) for their expert assistance with the mouse xenograft experiments as well as Richelle Cutler-Strom and Joe Ouadah (FHCRC) for technical support for in vitro work. We acknowledge both Donald K. Hamlin and D. Scott Wilbur (University of Washington) for providing the streptavidin-conjugated antibody. We thank Dave McDonald in the Scientific Imaging core facility (FHCRC) for assistance in acquiring the microscopy images and Wilo Dietrich for graphics support. We acknowledge helpful discussions with Bilal Ghosn and John Wilson (University of Washington).

\section{CONFLICTS OF INTEREST}

O.W. Press and P.S. Stayton are co-founders and consultants for PhaseRx Inc., a company that has licensed similar drug delivery technology, however this work was conducted independently of PhaseRx Inc. under NIH and WSLSDF support. None of the other authors have potential conflicts of interest.

\section{GRANT SUPPORT}

This research was funded by the following grants: NIH 5R01EB002991-07 (P.S. Stayton, O.W. Press); Washington State Life Sciences Discovery Fund
(WSLSDF) \#2496490 (P.S. Stayton, O.W. Press); NCI 1K08CA163603-01A1 (M.C. Palanca-Wessels); the Wayne D. Kuni \& Joan E. Kuni Foundation and the Kuni family through the 3725 Fund of the Oregon Community Foundation (M.C. Palanca-Wessels); NCI P50 CA083636, Pacific Ovarian Cancer Research Consortium (M.C. Palanca-Wessels); Breast Cancer Research Foundation (M.F. Press); Adelson Medical Research Foundation (M.F. Press).

\section{REFERENCES}

1. Castanotto D, Rossi JJ. The promises and pitfalls of RNA-interference-based therapeutics. Nature. 2009; 457:426-433.

2. de Fougerolles A, Vornlocher HP, Maraganore J, Lieberman J. Interfering with disease: a progress report on siRNA-based therapeutics. Nature reviews Drug discovery. 2007; 6:443-453.

3. Davis ME, Zuckerman JE, Choi CH, Seligson D, Tolcher A, Alabi CA, Yen Y, Heidel JD, Ribas A. Evidence of RNAi in humans from systemically administered siRNA via targeted nanoparticles. Nature. 2010; 464:1067-1070.

4. Tabernero J, Shapiro GI, LoRusso PM, Cervantes A, Schwartz GK, Weiss GJ, Paz-Ares L, Cho DC, Infante JR, Alsina M, Gounder MM, Falzone R, Harrop J, et al. Firstin-humans trial of an RNA interference therapeutic targeting VEGF and KSP in cancer patients with liver involvement. Cancer discovery. 2013; 3:406-417.

5. Bunn PA, Jr., Helfrich B, Soriano AF, Franklin WA, Varella-Garcia M, Hirsch FR, Baron A, Zeng C, Chan DC. Expression of Her-2/neu in human lung cancer cell lines by immunohistochemistry and fluorescence in situ hybridization and its relationship to in vitro cytotoxicity by trastuzumab and chemotherapeutic agents. Clinical cancer research. 2001; 7:3239-3250.

6. Hudis CA. Trastuzumab--mechanism of action and use in clinical practice. N Engl J Med. 2007; 357:39-51.

7. Kim SY, Kim HP, Kim YJ, Oh do Y, Im SA, Lee D, Jong HS, Kim TY, Bang YJ. Trastuzumab inhibits the growth of human gastric cancer cell lines with HER2 amplification synergistically with cisplatin. International journal of oncology. 2008; 32:89-95.

8. Menard S, Pupa SM, Campiglio M, Tagliabue E. Biologic and therapeutic role of HER2 in cancer. Oncogene. 2003; 22:6570-6578.

9. Tuefferd M, Couturier J, Penault-Llorca F, VincentSalomon A, Broet P, Guastalla JP, Allouache D, Combe M, Weber B, Pujade-Lauraine E, Camilleri-Broet S. HER2 status in ovarian carcinomas: a multicenter GINECO study of 320 patients. PLoS One. 2007; 2:e1138.

10. Bang YJ, Van Cutsem E, Feyereislova A, Chung HC, Shen L, Sawaki A, Lordick F, Ohtsu A, Omuro Y, Satoh T, Aprile G, Kulikov E, Hill J, et al. Trastuzumab in 
combination with chemotherapy versus chemotherapy alone for treatment of HER2-positive advanced gastric or gastrooesophageal junction cancer (ToGA): a phase 3, open-label, randomised controlled trial. Lancet (London, England). 2010; 376:687-697.

11. Barrajon-Catalan E, Menendez-Gutierrez MP, Falco A, Carrato A, Saceda M, Micol V. Selective death of human breast cancer cells by lytic immunoliposomes: Correlation with their HER2 expression level. Cancer letters. 2010; 290:192-203.

12. Mahmud H, Dalken B, Wels WS. Induction of programmed cell death in ErbB2/HER2-expressing cancer cells by targeted delivery of apoptosis-inducing factor. Molecular cancer therapeutics. 2009; 8:1526-1535.

13. Wang F, Ren J, Qiu XC, Wang LF, Zhu Q, Zhang YQ, Huan Y, Meng YL, Yao LB, Chen SY, Xu YM, Yang AG. Selective cytotoxicity to HER2-positive tumor cells by a recombinant e23sFv-TD-tBID protein containing a furin cleavage sequence. Clinical cancer research. 2010; 16:2284-2294.

14. Dou S, Yao YD, Yang XZ, Sun TM, Mao CQ, Song EW, Wang J. Anti-Her2 single-chain antibody mediated DNMTs-siRNA delivery for targeted breast cancer therapy. Journal of controlled release. 2012; 161:875-883.

15. Thiel KW, Hernandez LI, Dassie JP, Thiel WH, Liu X, Stockdale KR, Rothman AM, Hernandez FJ, McNamara JO, 2nd, Giangrande PH. Delivery of chemo-sensitizing siRNAs to HER2+-breast cancer cells using RNA aptamers. Nucleic acids research. 2012; 40:6319-6337.

16. Han H, Davis ME. Single-antibody, targeted nanoparticle delivery of camptothecin. Molecular pharmaceutics. 2013; 10:2558-2567.

17. Krop IE, LoRusso P, Miller KD, Modi S, Yardley D, Rodriguez G, Guardino E, Lu M, Zheng M, Girish S, Amler L, Winer EP, Rugo HS. A phase II study of trastuzumab emtansine in patients with human epidermal growth factor receptor 2-positive metastatic breast cancer who were previously treated with trastuzumab, lapatinib, an anthracycline, a taxane, and capecitabine. Journal of clinical oncology. 2012; 30:3234-3241.

18. Bookman MA, Darcy KM, Clarke-Pearson D, Boothby RA, Horowitz IR. Evaluation of monoclonal humanized anti-HER2 antibody, trastuzumab, in patients with recurrent or refractory ovarian or primary peritoneal carcinoma with overexpression of HER2: a phase II trial of the Gynecologic Oncology Group. Journal of clinical oncology. 2003; 21:283-290.

19. Palanca-Wessels MC CA, Cutler-Strom R, Lee F, Berguig GY, Stayton PS, Press OW. Anti-CD22 Antibody Targeting of $\mathrm{pH}$-Responsive Micelles Enhances Small Interfering RNA Delivery and Gene Silencing in Lymphoma Cells. Mol Ther. 2011; 19:1529-1537.

20. Vaughan S, Coward JI, Bast RC, Jr., Berchuck A, Berek JS, Brenton JD, Coukos G, Crum CC, Drapkin R, Etemadmoghadam D, Friedlander M, Gabra H, Kaye
$\mathrm{SB}$, et al. Rethinking ovarian cancer: recommendations for improving outcomes. Nature reviews Cancer. 2011; 11:719-725.

21. Williams J, Lucas PC, Griffith KA, Choi M, Fogoros S, Hu YY, Liu JR. Expression of Bcl-xL in ovarian carcinoma is associated with chemoresistance and recurrent disease. Gynecol Oncol. 2005; 96:287-295.

22. Wong M, Tan N, Zha J, Peale FV, Yue P, Fairbrother WJ, Belmont LD. Navitoclax (ABT-263) reduces $\mathrm{Bcl}-\mathrm{x}(\mathrm{L})$-mediated chemoresistance in ovarian cancer models. Molecular cancer therapeutics. 2012; 11:1026-1035.

23. Al Zaid Siddiquee K, Turkson J. STAT3 as a target for inducing apoptosis in solid and hematological tumors. Cell research. 2008; 18:254-267.

24. Commisso C, Davidson SM, Soydaner-Azeloglu RG, Parker SJ, Kamphorst JJ, Hackett S, Grabocka E, Nofal M, Drebin JA, Thompson CB, Rabinowitz JD, Metallo CM, Vander Heiden MG, et al. Macropinocytosis of protein is an amino acid supply route in Ras-transformed cells. Nature. 2013; 497:633-637.

25. Lundy BB, Convertine A, Miteva M, Stayton PS. Neutral polymeric micelles for RNA delivery. Bioconjugate chemistry. 2013; 24:398-407.

26. Rettig GR, Behlke MA. Progress toward in vivo use of siRNAs-II. Molecular therapy. 2012; 20:483-512.

27. Judge AD, Robbins M, Tavakoli I, Levi J, Hu L, Fronda A, Ambegia E, McClintock K, MacLachlan I. Confirming the RNAi-mediated mechanism of action of siRNA-based cancer therapeutics in mice. J Clin Invest. 2009; 119:661-673.

28. Abrams MT, Koser ML, Seitzer J, Williams SC, DiPietro MA, Wang W, Shaw AW, Mao X, Jadhav V, Davide JP, Burke PA, Sachs AB, Stirdivant SM, et al. Evaluation of efficacy, biodistribution, and inflammation for a potent siRNA nanoparticle: effect of dexamethasone co-treatment. Molecular therapy. 2010; 18:171-180.

29. Hassan R, Bera T, Pastan I. Mesothelin: a new target for immunotherapy. Clinical cancer research. 2004; 10:3937-3942.

30. James SE, Orgun NN, Tedder TF, Shlomchik MJ, Jensen MC, Lin Y, Greenberg PD, Press OW. Antibody-mediated B-cell depletion before adoptive immunotherapy with $\mathrm{T}$ cells expressing CD20-specific chimeric T-cell receptors facilitates eradication of leukemia in immunocompetent mice. Blood. 2009; 114:5454-5463.

31. Park JM, Yang X, Park JJ, Press OW, Press MF. Assessment of novel anti-p185HER-2 monoclonal antibodies for internalization-dependent therapies. Hybridoma. 1999; 18:487-495.

32. Hylarides MD, Mallett RW, Meyer DL. A robust method for the preparation and purification of antibody/streptavidin conjugates. Bioconjugate chemistry. 2001; 12:421-427.

33. Pagel JM, Hedin N, Subbiah K, Meyer D, Mallet R, Axworthy D, Theodore LJ, Wilbur DS, Matthews DC, Press OW. Comparison of anti-CD20 and anti-CD45 antibodies 
for conventional and pretargeted radioimmunotherapy of B-cell lymphomas. Blood. 2003; 101:2340-2348.

34. Livak KJ, Schmittgen TD. Analysis of relative gene expression data using real-time quantitative PCR and the 2(-Delta Delta C(T)) Method. Methods. 2001; 25:402-408.

35. Lasham A, Herbert M, Coppieters 't Wallant N, Patel R, Feng S, Eszes M, Cao H, Reid G. A rapid and sensitive method to detect siRNA-mediated mRNA cleavage in vivo using 5' RACE and a molecular beacon probe. Nucleic acids research. 2010; 38:e19.
36. Berglund SR, Schwietert CW, Jones AA, Stern RL, Lehmann J, Goldberg Z. Optimized methodology for sequential extraction of RNA and protein from small human skin biopsies. The Journal of investigative dermatology. 2007; 127:349-353.

37. Kim SH, Jeong JH, Lee SH, Kim SW, Park TG. Local and systemic delivery of VEGF siRNA using polyelectrolyte complex micelles for effective treatment of cancer. Journal of controlled release. 2008; 129:107-116. 